\title{
Space-Time Domain Decomposition with Finite Volumes for Porous Media Applications
}

\author{
Paul-Marie Berthe ${ }^{1}$, Caroline Japhet ${ }^{2}$, and Pascal Omnes ${ }^{1}$
}

\section{Introduction}

In the context of simulating flow and transport in porous media (e.g. for the assessment of nuclear waste repository safety), two main challenges must be taken into account : the heterogeneity of the medium with physical properties ranging over several orders of magnitude, and widely differing space-time scales. Solving these features accurately requires very fine meshes or well-adapted and highly nonconforming meshes. On the one hand, one possible approach is to use non-overlapping domain decomposition which leads to efficient parallel algorithms with local adaptation in both space and time. The Optimized Schwarz Waveform Relaxation method (OSWR) [3, 2] with the Discontinuous Galerkin (DG) scheme in time [4] is a solution procedure which allows local time stepping. On the other hand, the finite volume schemes of DDFV type (Discrete Duality Finite Volumes) for diffusion problems [5] allow highly nonconforming meshes. Finally, [6] presents a strategy which is well adapted to domain decomposition for coupling upwind discretization of the convection with diffusion in the context of a finite volume method. In this paper, we extend the OSWR method to the DDFV scheme for advection-diffusion problems, using the strategy of [6]. The method is proven to be well posed and we prove the convergence of the iterative algorithm.

We consider the following transport equation in a porous medium :

$$
\begin{aligned}
& \mathscr{L} c=\omega \partial_{t} c-\nabla \cdot(\boldsymbol{K} \nabla c-\boldsymbol{b} c)=f, \quad \text { in } \Omega \times(0, T), \\
& c(., 0)=c_{0}, \text { in } \Omega \text {, }
\end{aligned}
$$

where $\Omega$ is an open bounded polygonal subset of $\mathbb{R}^{2}, c$ is the concentration (e.g. of radionuclides) and $f$ the source term. Equation (1) is supplemented with homogeneous Dirichlet boundary conditions. We assume that $\Omega$ is decomposed into non-overlapping subdomains. For the sake of simplicity, we present the method in the case of two polygonal subdomains $\Omega_{L}$ and $\Omega_{R}$ with interface $\Gamma:=\partial \Omega_{L} \cap \partial \Omega_{R}$ (the method can be extended to the many subdomain case). We assume that the possible discontinuities of the porosity coefficient $\omega$, the tangential component of the advection velocity $\boldsymbol{b}$ and the anisotropic diffusion matrix $\boldsymbol{K}$ are along $\Gamma$. In the sequel, the subscripts and superscripts $L$ (resp. $R$ ) refer to $\Omega_{L}\left(\operatorname{resp} . \Omega_{R}\right)$.

${ }^{1}$ CEA, DEN, DM2S-STMF, F-91191 Gif-sur-Yvette Cedex, France. Université Paris 13, LAGA, F-93430, Villetaneuse, France. e-mail: \{berthe\} \{omnes\} @math.univ-paris13. fr

2 Université Paris 13, LAGA, UMR 7539, F-93430, Villetaneuse, France. INRIA ParisRocquencourt, BP 105, 78153 Le Chesnay, France, e-mail: Caroline. Japhet@inria.fr 
The initial problem (1) is equivalent to a system of subproblems defined on $\Omega_{L}$ and $\Omega_{R}$ with the following physical transmission conditions on $\Gamma:[c]_{\Gamma}=0$ and $[(\boldsymbol{K} \nabla c-\boldsymbol{b} c) \cdot \boldsymbol{n}]_{\Gamma}=0$, where $[v]_{\Gamma}$ denotes the jump of $v$ through $\Gamma$ and $\boldsymbol{n}$ a normal vector to $\Gamma$. These interface conditions can also be written, through Robin interface operators $\mathscr{B}_{L}$ and $\mathscr{B}_{R}$, under the equivalent form

$$
\begin{gathered}
{\left[\mathscr{B}_{L} c\right]_{\Gamma}=\left[\mathscr{B}_{R} c\right]_{\Gamma}=0,} \\
\text { with } \quad \mathscr{B}_{L}=(\boldsymbol{K} \nabla c-\boldsymbol{b c}) \cdot \boldsymbol{n}_{L}+\lambda_{L} \quad, \quad \mathscr{B}_{R}=(\boldsymbol{K} \nabla c-\boldsymbol{b} c) \cdot \boldsymbol{n}_{R}+\lambda_{R},
\end{gathered}
$$

where $\boldsymbol{n}_{L}$ (resp. $\boldsymbol{n}_{R}$ ) is the outward normal to $\Omega_{L}$ (resp. $\Omega_{R}$ ) and $\lambda_{L}\left(\right.$ resp. $\lambda_{R}$ ) a strictly positive function in $L^{\infty}(\Gamma)$.

Then, an OSWR algorithm [3, 2] for solving problem (1) is:

$$
\left\{\begin{array} { l l } 
{ \mathscr { L } c _ { L } ^ { ( \ell + 1 ) } = f } & { \text { in } \Omega _ { L } \times ( 0 , T ) } \\
{ c _ { L } ^ { ( \ell + 1 ) } ( . , 0 ) = c _ { 0 } } & { \text { in } \Omega _ { L } } \\
{ \mathscr { B } _ { L } c _ { L } ^ { ( \ell + 1 ) } = \mathscr { B } _ { L } c _ { R } ^ { ( \ell ) } \text { on } \Gamma \times ( 0 , T ) }
\end{array} \quad \left\{\begin{array}{ll}
\mathscr{L} c_{R}^{(\ell+1)}=f & \text { in } \Omega_{R} \times(0, T) \\
c_{R}^{(\ell+1)}(., 0)=c_{0} & \text { in } \Omega_{R} \\
\mathscr{B}_{R} c_{R}^{(\ell+1)}=\mathscr{B}_{R} c_{L}^{(\ell)} & \text { on } \Gamma \times(0, T)
\end{array}\right.\right.
$$

where $\lambda_{L}$ and $\lambda_{R}$ optimize the convergence factor of (4), see [2, 8, 9].

In Section 2, we present the DDFV scheme for the advection-diffusion problem in the global domain $\Omega$. Then, in Section 3, we describe the multidomain DDFV scheme. Section 4 is devoted to the OSWR algorithm for the DDFV scheme. Finally in Section 5, we present numerical results.

\section{The DDFV scheme for advection-diffusion problems}

In this part, we present the DDFV scheme for Problem (1). This scheme uses unknowns at the centers of the cells of a primal mesh and at their vertices. These vertices are considered as the centers of dual cells, obtained by joining the centers of the surrounding primal cells through the edge midpoints. This construction is sufficiently general to be able to treat non-conforming meshes, see Fig. 1 (left) where the primal (resp. dual) nodes are in black (resp. red), and $T_{i_{1}}$ (resp. $P_{k_{1}}$ ) is an example of primal (resp. dual) cell. Using these supplementary vertex unknowns is the price to pay to be able to use arbitrary meshes [5]. We split $(0, T)$ into time intervals $I_{n}:=\left(t_{n-1}, t_{n}\right)$ and define $\Delta t_{n}:=t_{n}-t_{n-1}$. We denote by $c_{i_{1}}^{n}\left(\right.$ resp. $\left.c_{k_{1}}^{n}\right)$ an approximation of $c$ at time $t_{n}$ in the cell $T_{i_{1}}$ (resp. $P_{k_{1}}$ ). Restricting the presentation to the lowest order DG scheme in time, equation (1) can be discretized on each time interval and on each primal cell $T_{i_{1}}$ by

$$
\omega_{i_{1}} \frac{c_{i_{1}}^{n}-c_{i_{1}}^{n-1}}{\Delta t_{n}}-\frac{1}{\left|T_{i_{1}}\right|} \sum_{A_{j} \subset \partial T_{i_{1}}}\left|A_{j}\right| F_{i_{1} j}^{n}=f_{i_{1}}^{n}:=\frac{1}{\Delta t_{n}\left|T_{i_{1}}\right|} \int_{I_{n}} \int_{T_{i_{1}}} f(\boldsymbol{x}, t) d \boldsymbol{x} d t
$$

and on each inner dual cell $P_{k_{1}}$ by 

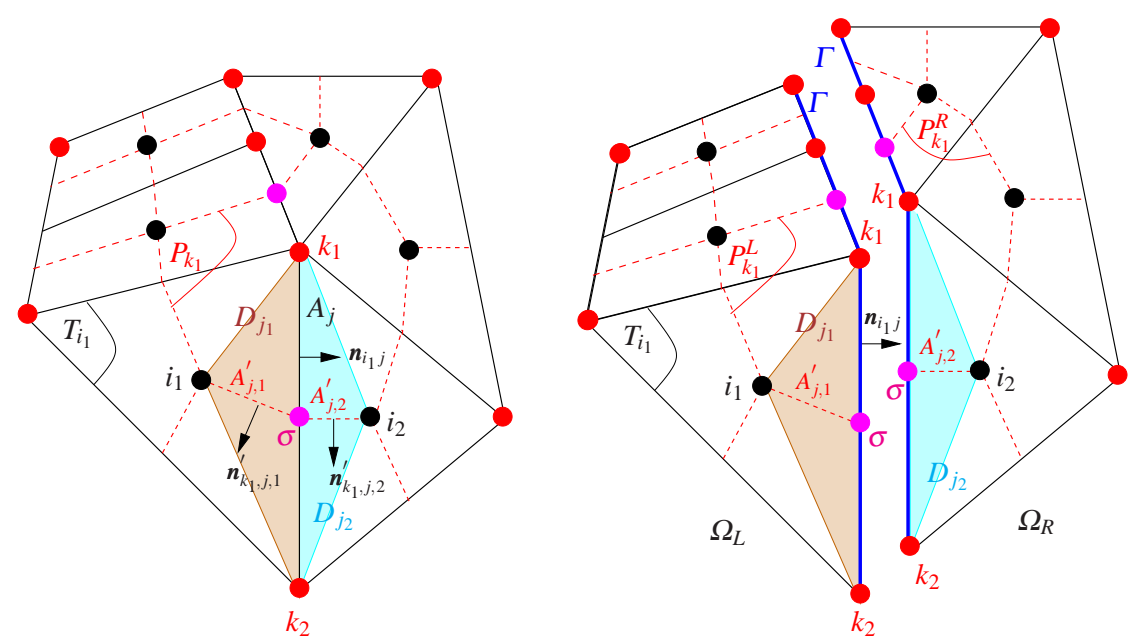

Fig. 1 DDFV primal (solid lines), dual (dashed lines) and half-diamond cells (filled triangles): interior (left) and interface (right) cells.

$$
\omega_{k_{1}} \frac{c_{k_{1}}^{n}-c_{k_{1}}^{n-1}}{\Delta t_{n}}-\frac{1}{\left|P_{k_{1}}\right|} \sum_{A_{j, \alpha}^{\prime} \subset \partial P_{k_{1}}}\left|A_{j, \alpha}^{\prime}\right| F_{k_{1} j, \alpha}^{n}=f_{k_{1}}^{n}:=\frac{1}{\Delta t_{n}\left|P_{k_{1}}\right|} \int_{I_{n}} \int_{P_{k_{1}}} f(\boldsymbol{x}, t) d \boldsymbol{x} d t .
$$

In (6), the subscript $\alpha \in\{1,2\}$ refers to the local numbering $i_{1}, i_{2}$, and $\omega_{k_{1}}$ is defined by

$$
\left|P_{k_{1}}\right| \omega_{k_{1}}=\left|P_{k_{1}} \cap \Omega_{L}\right| \omega_{k_{1}}^{L}+\left|P_{k_{1}} \cap \Omega_{R}\right| \omega_{k_{1}}^{R} .
$$

In order to lighten the notations, we leave out the exponents $n$ in this section.

For any primal edge $A_{j}=\left[k_{1} k_{2}\right]$ and its associated dual edges $A_{j, \alpha}^{\prime}$, the fluxes $F_{i_{1} j}$ and $F_{k_{1} j, \alpha}$ are sums of a diffusive and a convective contribution. The diffusive part is evaluated as in [5] using a gradient defined by two directions, on each triangle $k_{1} i_{\alpha} k_{2}=: D_{j, \alpha}$ (also called "half-diamond cell"), see Fig. 1 (left):

$$
\left\{\begin{array}{l}
\left(\nabla_{h} c\right)_{i_{\alpha} j} \cdot \overrightarrow{i_{\alpha} \sigma}=c_{\sigma}-c_{i_{\alpha}} \\
\left(\nabla_{h} c\right)_{i_{\alpha} j} \cdot \overrightarrow{k_{1} k_{2}}=c_{k_{2}}-c_{k_{1}}
\end{array}\right.
$$

where $\sigma$ is the midpoint of $A_{j}$. Formulas (8) are equivalent to

$$
\left(\nabla_{h} c\right)_{i_{\alpha} j}=\frac{1}{\left|D_{j, \alpha}\right|}\left(\left(c_{k_{2}}-c_{k_{1}}\right)\left|A_{j, \alpha}^{\prime}\right| \boldsymbol{n}_{k_{1} j, \alpha}^{\prime}+\left(c_{\sigma}-c_{i_{\alpha}}\right)\left|A_{j}\right| \boldsymbol{n}_{i_{1} j}\right),
$$

where $\boldsymbol{n}_{i_{1} j}$ is the outward normal to $T_{i_{1}}$ on $A_{j}$ and $\boldsymbol{n}_{k_{1} j, \alpha}^{\prime}$ the outward normal to $P_{k_{1}}$ on $A_{j, \alpha}^{\prime}$. The unknown $c_{\sigma}$ is introduced both to deal with possibly discontinuous tensors $\boldsymbol{K}$ and to be able to write a local discretization adapted to domain decomposition, as will be shown in Section 3. The gradient $\left(\nabla_{h} c\right)_{i_{\alpha} j}$ is used in the diffusive part of $F_{i_{\alpha} j}$ and in the diffusive part of $F_{k_{1} j, \alpha}$ and $F_{k_{2} j, \alpha}$. Let us denote by $[a]^{+}$and $[a]^{-}$the 
positive and negative part of $a$ such that $a=[a]^{-}+[a]^{+}$. The convective part of the flux on the primal mesh is discretized with an upwind scheme which is local to the half-diamond cell $D_{j, \alpha}$ :

$$
(\boldsymbol{b} \boldsymbol{c} \cdot \boldsymbol{n})_{i_{\alpha} j}:=\left[(\boldsymbol{b} \cdot \boldsymbol{n})_{i_{\alpha} j}\right]^{+} c_{i_{\alpha}}+\left[(\boldsymbol{b} \cdot \boldsymbol{n})_{i_{\alpha} j}\right]^{-} c_{\sigma} .
$$

This upwinding using $c_{\sigma}$ ensures that the discretization of the convection flux is local to a subdomain. This is the idea borrowed from [6]. On the dual mesh, we use a standard upwind scheme:

$$
\left(\boldsymbol{b} c \cdot \boldsymbol{n}^{\prime}\right)_{k_{1} j, \alpha}:=\left[\boldsymbol{b}_{j, \alpha} \cdot \boldsymbol{n}_{k_{1} j, \alpha}^{\prime}\right]^{+} c_{k_{1}}+\left[\boldsymbol{b}_{j, \alpha} \cdot \boldsymbol{n}_{k_{1} j, \alpha}^{\prime}\right]^{-} c_{k_{2}} .
$$

In (10), $(\boldsymbol{b} \cdot \boldsymbol{n})_{i_{\alpha} j}$ is defined by (recall that $\boldsymbol{b} \cdot \boldsymbol{n}$ is continuous through primal edges)

$$
(\boldsymbol{b} \cdot \boldsymbol{n})_{i_{\alpha} j}:=\frac{1}{\left|A_{j}\right|} \int_{A_{j}} \boldsymbol{b} \cdot \boldsymbol{n}_{i_{\alpha j}}(\xi) d \xi
$$

In (11), $\boldsymbol{b}_{j, \alpha}$ is the mean-value of $\boldsymbol{b}$ over $A_{j, \alpha}^{\prime}$. The fluxes are then defined as follows:

$$
\begin{aligned}
F_{i_{\alpha} j} & :=\left[\boldsymbol{K}_{i_{\alpha} j}\left(\nabla_{h} c\right)_{i_{\alpha} j}\right] \cdot \boldsymbol{n}_{i_{\alpha} j}-(\boldsymbol{b} c \cdot \boldsymbol{n})_{i_{\alpha} j}, \\
F_{k_{1} j, \alpha} & :=\left[\boldsymbol{K}_{j, \alpha}\left(\nabla_{h} c\right)_{i_{\alpha j} j}\right] \cdot \boldsymbol{n}_{k_{1} j, \alpha}^{\prime}-\left(\boldsymbol{b} c \cdot \boldsymbol{n}^{\prime}\right)_{k_{1 j}, \alpha} .
\end{aligned}
$$

In (13) and (14), $\boldsymbol{K}_{i_{\alpha} j}$ and $\boldsymbol{K}_{j, \alpha}$ are the mean-values of $\boldsymbol{K}_{\mid T_{i \alpha}}$ over $A_{j}$ and $A_{j, \alpha}^{\prime}$, respectively (we recall that $\boldsymbol{K}$ may be discontinuous through primal edges $A_{j}$ ). In order to complete the definition of the scheme, we still need an equation for each $c_{\sigma}$, and one equation for each boundary dual cell. If $\sigma$ is not on $\partial \Omega, c_{\sigma}$ is eliminated by requiring the flux conservation through the common interface $\partial T_{i_{1}} \cap \partial T_{i_{2}}$ :

$$
F_{i_{1} j}+F_{i_{2} j}=0 .
$$

Formula (15) defines a unique $c_{\sigma}$ that we replace in (9) and (10). For nodes $\sigma$ and $k$ located on the Dirichlet boundary, we set

$$
c_{\sigma}=c_{k}=0, \forall \sigma \in \partial \Omega, \forall k \in \partial \Omega \text {. }
$$

Theorem 1. We suppose that $\nabla \cdot \boldsymbol{b} \geq 0$ and that $\boldsymbol{K}$ is a bounded, uniformly definite positive matrix. Then, the discrete convection-diffusion problem in the global domain $\Omega$, defined by formulas (5) to (16) is well-posed.

\section{The multidomain DDFV scheme}

In this part we describe the local DDFV scheme in a subdomain together with the discretization of the Robin conditions (2)-(3). 
The subdomain scheme is not modified for primal cells : we still use (5) and (13) with the superscript ${ }^{L}$ (resp. ${ }^{R}$ ) for $\Omega_{L}$ (resp. $\Omega_{R}$ ), $c_{\sigma}^{L, R}=0$ on the Dirichlet boundary and (15) when $\sigma$ is not on $\partial \Omega$ nor on $\Gamma$. Moreover, when $\sigma$, midpoint of a primal edge $A_{j}$, is on $\Gamma$, we discretize the Robin conditions (2)-(3) on $A_{j}$ by

$$
\begin{aligned}
& F_{i_{1} j}^{L, n}+\lambda_{L, j} c_{\sigma}^{L, n}=-F_{i_{2} j}^{R, n}+\lambda_{L, j} c_{\sigma}^{R, n}, \\
& F_{i_{2} j}^{R, n}+\lambda_{R, j} c_{\sigma}^{R, n}=-F_{i_{1} j}^{L, n}+\lambda_{R, j} c_{\sigma}^{L, n},
\end{aligned}
$$

where $\lambda_{L, j}$ and $\lambda_{R, j}$ are discrete counterparts of $\lambda_{L}$ and $\lambda_{R}$ defined on each primal edge $A_{j}$. In (17) and (18), we use the convention that $i_{1}$ is in $\Omega_{L}$ and $i_{2}$ in $\Omega_{R}$. We remark that (17)-(18) are equivalent to $c_{\sigma}^{R, n}=c_{\sigma}^{L, n}$ and $F_{i_{1} j}^{L, n}+F_{i_{2} j}^{R, n}=0$.

On interior dual cells, the scheme is not modified: we still use (6) with the superscript ${ }^{L}$ (resp. ${ }^{R}$ ) for $\Omega_{L}$ (resp. $\Omega_{R}$ ). Moreover, $c_{k}^{L, R}=0$ if $k$ is a node located on the Dirichlet boundary. Finally, if $k_{1}$ belongs to $\Gamma \backslash \partial \Omega$, then we denote by $P_{k_{1}}^{L}$ (resp. $P_{k_{1}}^{R}$ ) the boundary dual cell in $\Omega_{L}$ (resp. $\Omega_{R}$ ) to which $k_{1}$ is associated (see Fig. 1, right). The cell $P_{k_{1}}^{L}$ (resp. $P_{k_{1}}^{R}$ ) has two types of edges: the edges $A_{j, \alpha}^{\prime}$ that belong to $\partial P_{k_{1}}^{L} \backslash \Gamma$ (resp. $\partial P_{k_{1}}^{R} \backslash \Gamma$ ) and the edges on $\partial P_{k_{1}}^{L} \cap \Gamma$ (resp. $\partial P_{k_{1}}^{R} \cap \Gamma$ ). Integrating (1) on $P_{k_{1}}^{L}$ and over $I_{n}$ yields the approximation

$$
\omega_{k_{1}}^{L}\left|P_{k_{1}}^{L}\right|\left(\frac{c_{k_{1}}^{L n}-c_{k_{1}}^{L n-1}}{\Delta t_{n}}\right)-\sum_{A_{j, \alpha}^{\prime} \subset \partial P_{k_{1}}^{L}}\left|A_{j, \alpha}^{\prime}\right| F_{k_{1} j, \alpha}^{n}-\left|\partial P_{k_{1}}^{L} \cap \Gamma\right| F_{k_{1}, \Gamma}^{L n}=\left|P_{k_{1}}^{L}\right| f_{k_{1}}^{L n},
$$

where $F_{k_{1}, \Gamma}^{L n}$ is an approximation of $\frac{1}{\Delta t_{n}\left|\partial P_{k_{1}}^{L} \cap \Gamma\right|} \int_{I_{n}} \int_{\partial P_{k_{1}}^{L} \cap \Gamma}(\boldsymbol{K} \nabla c-\boldsymbol{b} c) \cdot \boldsymbol{n}_{L}$ and $f_{k_{1}}^{L n}$ is defined similarly to $f_{k_{1}}^{n}$ in (6) in which $P_{k_{1}}$ is replaced by $P_{k_{1}}^{L}$. In the same way, we define $F_{k_{1}, \Gamma}^{R n}$ and $f_{k_{1}}^{R n}$, and we obtain the following approximation of (1) on $P_{k_{1}}^{R}$

$$
\omega_{k_{1}}^{R}\left|P_{k_{1}}^{R}\right|\left(\frac{c_{k_{1}}^{R n}-c_{k_{1}}^{R n-1}}{\Delta t_{n}}\right)-\sum_{A_{j, \alpha}^{\prime} \subset \partial P_{k_{1}}^{R}}\left|A_{j, \alpha}^{\prime}\right| F_{k_{1} j, \alpha}^{n}-\left|\partial P_{k_{1}}^{R} \cap \Gamma\right| F_{k_{1}, \Gamma}^{R n}=\left|P_{k_{1}}^{R}\right| f_{k_{1}}^{R n} .
$$

Equations (19) and (20) introduce new flux unknowns $F_{k_{1}, \Gamma}^{L n}$ and $F_{k_{1}, \Gamma}^{R n}$ which are related to the boundary unknowns $c_{k_{1}}^{L n}$ and $c_{k_{1}}^{R n}$ by the following dual approximations of the Robin boundary conditions (2)-(3)

$$
\begin{aligned}
& F_{k_{1}, \Gamma}^{L n}+\lambda_{L, k_{1}} c_{k_{1}}^{L n}=-F_{k_{1}, \Gamma}^{R n}+\lambda_{L, k_{1}} c_{k_{1}}^{R n}, \\
& F_{k_{1}, \Gamma}^{R n}+\lambda_{R, k_{1}} c_{k_{1}}^{R n}=-F_{k_{1}, \Gamma}^{L n}+\lambda_{R, k_{1}} c_{k_{1}}^{L n},
\end{aligned}
$$

where $\lambda_{L, k_{1}}$ and $\lambda_{R, k_{1}}$ are discrete counterparts of $\lambda_{L}$ and $\lambda_{R}$ defined on each dual intersection $\partial P_{k_{1}}^{L} \cap \Gamma=\partial P_{k_{1}}^{R} \cap \Gamma$. We remark that (21) and (22) are equivalent to $c_{k_{1}}^{L n}=c_{k_{1}}^{R n}$ and $F_{k_{1}, \Gamma}^{L n}+F_{k_{1}, \Gamma}^{R n}=0$. With these equalities for all time steps, adding (19) and (20) and using (7) yields (6) on $P_{k_{1}}=P_{k_{1}}^{L} \cup P_{k_{1}}^{R}$, the inner dual cell of the global domain $\Omega$. 
In order to study the well-posedness of the subdomain problems, we restrict ourselves to one subdomain, e.g. $\Omega_{L}$. Recalling that $(\boldsymbol{b} \cdot \boldsymbol{n})_{i_{\alpha} j}$ is defined by (12) and defining $(\boldsymbol{b} \cdot \boldsymbol{n})_{k_{1}}^{L}$ by

$$
(\boldsymbol{b} \cdot \boldsymbol{n})_{k_{1}, \Gamma}^{L}:=\frac{1}{\left|\partial P_{k_{1}}^{L} \cap \Gamma\right|} \int_{\partial P_{k_{1}}^{L} \cap \Gamma} \boldsymbol{b} \cdot \boldsymbol{n}_{L}(\boldsymbol{\xi}) d \boldsymbol{\xi},
$$

we can prove the following theorem

Theorem 2. Under the hypothesis of Theorem 1 , if $\lambda_{L, j}>\frac{1}{2}(\boldsymbol{b} \cdot \boldsymbol{n})_{i_{1} j}$ for all $j$ such that $A_{j} \subset \Gamma$ and if $\lambda_{L, k_{1}}>\frac{1}{2}(\boldsymbol{b} \cdot \boldsymbol{n})_{k_{1}, \Gamma}^{L}$ for all $k$ such that $\partial P_{k}^{L} \cap \Gamma \neq \emptyset$, then the discrete problem in $\Omega_{L}$, defined by formulas (5)-(6) and (13) to (16) with the superscript $^{L}$, formula (19) for boundary dual cells, and the Robin conditions

$$
\begin{aligned}
F_{i_{1} j}^{L, n}+\lambda_{L, j} c_{\sigma}^{L, n}=g_{j}^{L, n} & \left(\text { on primal edges } A_{j} \subset \Gamma\right) \\
F_{k_{1}, \Gamma}^{L n}+\lambda_{L, k_{1}} c_{k_{1}}^{L n}=g_{k_{1}}^{L, n} & \left(\text { on dual edges } \partial P_{k_{1}}^{L} \cap \Gamma\right),
\end{aligned}
$$

with $g_{j}^{L, n}$ and $g_{k_{1}}^{L, n}$ given real numbers, is well-posed.

\section{The Schwarz algorithm}

Let $S$ denote the superscript ${ }^{L}$ or ${ }^{R}$. The discrete Schwarz algorithm is defined as follows: let $\left(c_{i}^{S n(\ell)}, c_{k}^{S n(\ell)}, c_{\sigma}^{S n(\ell)}\right)$ and $\left(F_{i j}^{S n(\ell)}, F_{k j, \alpha}^{S n(\ell)}, F_{k, \Gamma}^{S n(\ell)}\right)$ be given approximations, at step $\ell$, of $c$ at nodes $i, k, \sigma$ and $(\boldsymbol{K} \nabla c-\boldsymbol{b} c) \cdot \boldsymbol{n}$ at edges $A_{j}, A_{j, \alpha}^{\prime}, \partial P_{k}^{S} \cap \Gamma$. Then we compute $\left(c_{i}^{S n(\ell+1)}, c_{k}^{S n(\ell+1)}, c_{\sigma}^{S n(\ell+1)}\right)$ and $\left(F_{i j}^{S n(\ell+1)}, F_{k j, \alpha}^{S n(\ell+1)}, F_{k, \Gamma}^{S n(\ell+1)}\right)$ as the solution of (5)-(6) and (13) to (16) with the superscript ${ }^{L}$ (resp. ${ }^{R}$ ), formula (19) (resp. (20)) and the following Robin conditions for interface primal and dual cells:

$$
\begin{aligned}
& F_{i_{1} j}^{L n(\ell+1)}+\lambda_{L, j} c_{\sigma}^{L n(\ell+1)}=-F_{i_{2} j}^{R n(\ell)}+\lambda_{L, j} c_{\sigma}^{R n(\ell)}, \\
& F_{k_{1}, \Gamma}^{L n(\ell+1)}+\lambda_{L, k_{1}} c_{k_{1}}^{L n(\ell+1)}=-F_{k_{1}, \Gamma}^{R n(\ell)}+\lambda_{L, k_{1}} c_{k_{1}}^{R n(\ell)}, \\
& F_{i_{2} j}^{R n(\ell+1)}+\lambda_{R, j} c_{\sigma}^{R n(\ell+1)}=-F_{i_{1} j}^{L n(\ell)}+\lambda_{R, j} c_{\sigma}^{L n(\ell)}, \\
& F_{k_{1}, \Gamma}^{R n(\ell+1)}+\lambda_{R, k_{1}} c_{k_{1}}^{R n(\ell+1)}=-F_{k_{1}, \Gamma}^{L n(\ell)}+\lambda_{R, k_{1}} c_{k_{1}}^{L n(\ell)} .
\end{aligned}
$$

Theorem 3. Under the hypothesis of Theorem 2, if $\lambda_{R, k_{1}}-\lambda_{L, k_{1}}-(\boldsymbol{b} \cdot \boldsymbol{n})_{k_{1}, \Gamma}^{L}=0$ for all $k$ such that $\partial P_{k}^{L} \cap \Gamma \neq \emptyset$ and if $\lambda_{R, j}-\lambda_{L, j}-(\boldsymbol{b} \cdot \boldsymbol{n})_{i_{1} j}=0$ for all $j$ such that $A_{j} \subset \Gamma$, then the discrete Schwarz algorithm converges to the solution of the discrete convection-diffusion problem in the domain $\Omega$, defined by formulas (5) to (16).

Remark 1. Following [8, 9], the Robin parameters are chosen in the form 


$$
\begin{gathered}
\lambda_{L, j}=\left(-(\boldsymbol{b} \cdot \boldsymbol{n})_{i_{1} j}+p_{L, j}\right) / 2, \lambda_{R, j}=\left((\boldsymbol{b} \cdot \boldsymbol{n})_{i_{1} j}+p_{R, j}\right) / 2, \\
\lambda_{L, k_{1}}=\left(-(\boldsymbol{b} \cdot \boldsymbol{n})_{k_{1}, \Gamma}^{L}+p_{L, k_{1}}\right) / 2, \lambda_{R, k_{1}}=\left((\boldsymbol{b} \cdot \boldsymbol{n})_{k_{1}, \Gamma}^{L}+p_{R, k_{1}}\right) / 2,
\end{gathered}
$$

where $p_{L, j}, p_{R, j}$ and $p_{L, k_{1}}, p_{R, k_{1}}$ are the primal and dual parameters which optimize the convergence factor of the continuous algorithm (4). This optimization is performed by a numerical minimization process. With the form given by (23)-(24), the hypothesis in Theorem 3 reduces to $p_{L, j}=p_{R, j}$ and $p_{L, k_{1}}=p_{R, k_{1}}$.

Remark 2. The scheme we proposed here is different from the one developed in [1]. On the other hand, it is shown independently in [7], using an analysis of the convergence factor at the discrete level, that our method leads to a faster convergence than the approach in [1]. In our simulations, we observed that using the optimized parameters at the discrete level does not improve significantly the convergence.

\section{Numerical Results}

Here, the Robin parameter for $\Omega_{L / R}$ is taken as the mean value of all $\lambda_{L / R, j}$ and $\lambda_{L / R, k_{1}}$ and is denoted $\lambda_{L / R}^{*}$. Moreover, $\boldsymbol{b} \cdot \boldsymbol{n}=0$ on $\Gamma$ in our tests, thus $\lambda_{L / R}^{*}=p^{*}$, the same value for all primal and dual $(L$ and $R)$ interface cells. Its discrete counterpart $p_{h}^{*}$ is obtainded in the same way but with an optimization of the discrete convergence factor, denoted $\rho_{h}$. We assume that $\boldsymbol{K}=v \boldsymbol{I}$ where $\boldsymbol{I}$ is the identity matrix.

In the first test case, we take $\Omega_{L}=(0,2.5) \times(0,5)$ and $\Omega_{R}=(2.5,5) \times(0,5)$, with $T=1, \omega_{L}=\omega_{R}=1, \boldsymbol{b}=\mathbf{0}, v_{\mid \Omega_{L}}=0.06$, and $v_{\mid \Omega_{R}}=1$. The mesh size and time step are $h=\frac{5}{100}$ and $\Delta t=\frac{1}{70}$ respectively. On Fig. 2 we show a section along the diagonal $\left(w_{m}, k_{m}\right)-\left(w_{M}, k_{M}\right)$ of $\rho_{h}$ (top left), where $\left(w_{m}, w_{M}\right) \times\left(k_{m}, k_{M}\right)$ is the frequencies interval over which $\rho_{h}$ is optimized, with $w_{m}=\frac{\pi}{T}, w_{M}=\frac{\pi}{\Delta t}, k_{m}=\frac{\pi}{5}, k_{M}=\frac{\pi}{h}$, and the error versus the number of iterations for the Schwarz algorithm (top right) with $p^{*}$ and $p_{h}^{*}$. We simulate directly the error equations, $f=0$ and use a random initial guess so that all the frequency components are present. We observe that using $p_{h}^{*}$ or $p^{*}$ give similar results. We also observe the equioscillation property [2] with $p_{h}^{*}$.

In the second test case, we take $\Omega_{L}=(0,0.5) \times(0,1)$ and $\Omega_{R}=(0.5,1) \times(0,1)$, with $T=1, \omega_{L}=0.2, \omega_{R}=1, v_{\mid \Omega_{L}}=0.005, v_{\mid \Omega_{R}}=0.01$, and a rotating velocity field $\boldsymbol{b}=\left(-\sin \left(\pi\left(y-\frac{1}{2}\right)\right) \cos \left(\pi\left(x-\frac{1}{2}\right)\right), \cos \left(\pi\left(y-\frac{1}{2}\right)\right) \sin \left(\pi\left(x-\frac{1}{2}\right)\right)\right)$. We take $h=$ $\frac{1}{100}$ and $\Delta t=\frac{1}{50}$. On Fig. 2 we show the computed solution at time $t=0.4$ (bottom left) and the error versus the number of iterations (bottom right) for different values of the Robin parameter $p$, taken constant along the interface. We take $f=0$ and a random initial guess. We observe that $p^{*}$ is close to the optimal numerical value. 

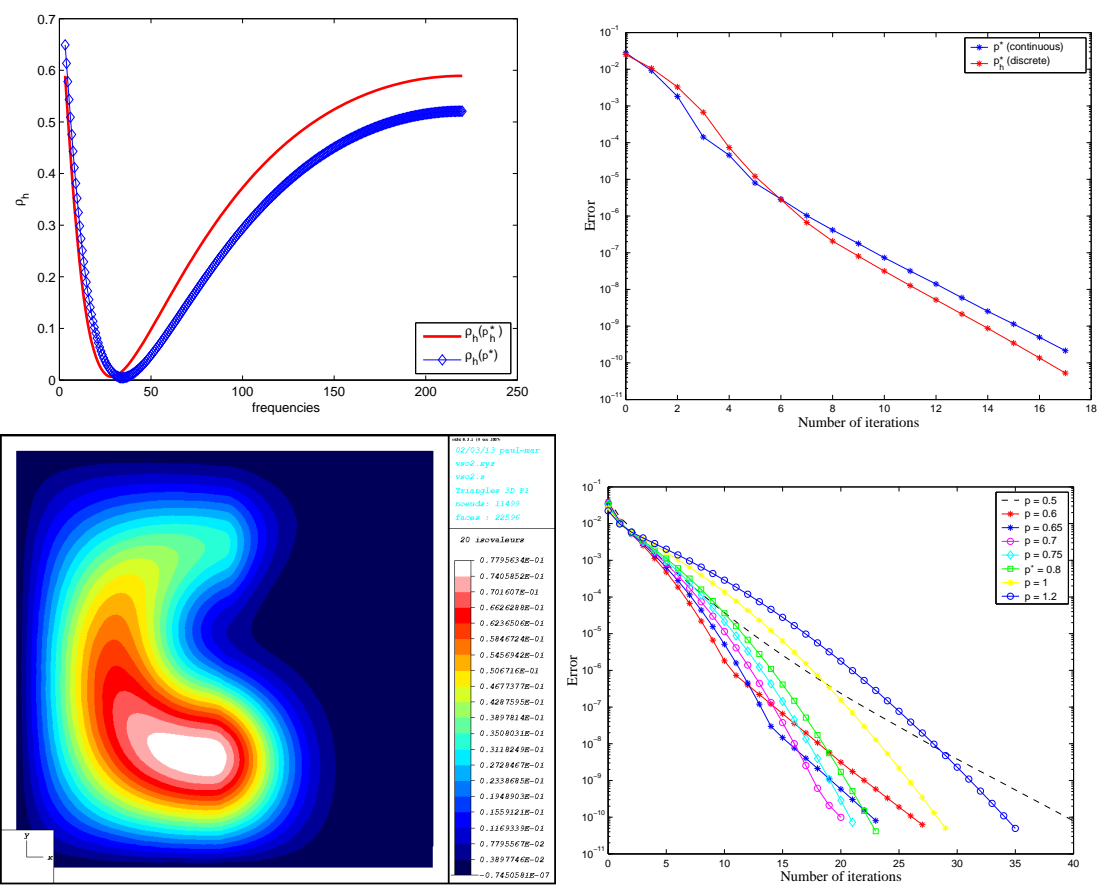

Fig. 2 Top: Discrete convergence factor (left) and error versus iterations (right), with $p^{*}$ and $p_{h}^{*}$. Bottom: solution at time $t=0.4$ (left) and error versus iterations (right) for different values of $p$.

\section{References}

1. Boyer, F., Hubert, F., Krell, S.: Non-overlapping Schwarz algorithm for solving 2D m-DDFV schemes. IMA Journal on Numerical Analysis 4 (30), 1062-1100 (2010)

2. Bennequin, D., Gander, M.J., Halpern, L.: A Homographic Best Approximation Problem with Application to Optimized Schwarz Waveform Relaxation. Math. of Comp. 78, 185-223 (2009)

3. Martin, V.: An optimized Schwarz waveform relaxation method for the unsteady convection diffusion equation in two dimensions. Appl. Numer. Math. 52, 401-428 (2005)

4. Halpern, L., Japhet, C., Szeftel, J.: Optimized Schwarz waveform relaxation and discontinuous Galerkin time stepping for heterogeneous problems. SIAM J. Numer. Anal. 50 (5), 2588-2611 (2012)

5. Domelevo, K., Omnes, P.: A finite volume method for the Laplace equation on almost arbitrary two-dimensional grids. Math. Model. Numer. Anal. 39, 1203-1249" (2005)

6. Haeberlein, F.: Time Space Domain Decomposition Methods for Reactive Transport - Application to CO2 Geological Storage. PhD thesis, University Paris XIII (2011)

7. Gander, M.J., Hubert, F., Krell, S.: Optimized Schwarz algorithms in the framework of DDFV schemes. In this proceedings.

8. Gander, M.J., Halpern, L., Kern, M.: A Schwarz waveform relaxation method for advectiondiffusion-reaction problems with discontinuous coefficients and non-matching grids. Domain Decomposition Methods in Science and Engineering, Lecture Notes in Computational Science and Engineering 55, 283-290. Springer (2007)

9. Halpern, L., Japhet, C., Omnes, P.: Nonconforming in time domain decomposition method for porous media applications. ECCOMAS CFD 2010, C.F. Pereira, A.Sequeira (Eds) (2010) 